\title{
Weak Solutions for a Fourth Order Degenerate Parabolic Equation
}

by

\author{
Changchun LIU and Jinyong GUO \\ Presented by Bogdan BOJARSKI
}

\begin{abstract}
Summary. We consider an initial-boundary value problem for a fourth order degenerate parabolic equation. Under some assumptions on the initial value, we establish the existence of weak solutions by the discrete-time method. The asymptotic behavior and the finite speed of propagation of perturbations of solutions are also discussed.
\end{abstract}

1. Introduction. This paper is concerned with a fourth order degenerate parabolic equation of the form

$$
\frac{\partial u}{\partial t}+\Delta\left(|\Delta u|^{p-2} \Delta u\right)+\lambda|u|^{p-2} u=0, \quad x \in \Omega, t>0, p>2,
$$

where $\lambda>0$ and $\Omega \subset \mathbb{R}^{N}$ is a bounded domain with smooth boundary.

On the basis of physical considerations, as usual the equation (1.1) is supplemented with the natural boundary conditions

$$
u=\Delta u=0, \quad x \in \partial \Omega, t>0,
$$

and the initial condition

$$
u(x, 0)=u_{0}(x), \quad x \in \Omega .
$$

The equation (1.1) is a typical higher order equation, which has a rich theoretical connotation. In the past years, there have been many contributions devoted to the $p$-biharmonic equation. Jiří Benedikt [1] studied the

2000 Mathematics Subject Classification: 35D05, 35B40, 35G30, 35K55. havior.

Key words and phrases: fourth order parabolic equation, existence, asymptotic be-

This work is supported by the Tianyuan Fund for Mathematics of China (No. $10526022)$. 
p-biharmonic equation

$$
\left(\left|u^{\prime \prime}\right|^{p-2} u^{\prime \prime}\right)^{\prime \prime}=\lambda|u|^{q-2} u,
$$

where $\lambda \in \mathbb{R}$ and $p, q>1$. He proved existence and uniqueness of a solution of the initial value problem. He also considered the equation with general Robin-type boundary conditions [2], and showed that every positive eigenvalue $\lambda$ is simple.

Pavel Drábek and Mitsuharu Ôtani [6] considered the equation

$$
\Delta\left(|\Delta u|^{p-2} \Delta u\right)=\lambda|u|^{p-2} u
$$

and proved that (1.4), (1.2) has a principal positive eigenvalue $\lambda_{1}$ which is simple and isolated.

Our equation resembles the $p$-Laplacian equation, but many methods used for the latter, like those based on the maximum principle, are no longer valid for this equation. Because of the degeneracy, the problem (1.1)-(1.3) does not admit classical solutions in general. So, we introduce weak solutions in the sense of the following

Definition. A function $u$ is said to be a weak solution of the problem (1.1)-(1.3) if the following conditions are satisfied:

1) $u \in L^{\infty}\left(0, T ; W_{0}^{2, p}(\Omega)\right) \cap C\left(0, T ; L^{2}(\Omega)\right), \partial u / \partial t \in L^{\infty}\left(0, T ; W^{-2, p^{\prime}}(\Omega)\right)$, where $p^{\prime}$ is the conjugate exponent of $p$.

2) For any $\varphi \in C_{0}^{\infty}\left(Q_{T}\right)$, where $Q_{T}=\Omega \times(0, T)$, the following integral equality holds:

$$
-\iint_{Q_{T}} u \frac{\partial \varphi}{\partial t} d x d t+\iint_{Q_{T}}|\Delta u|^{p-2} \Delta u \Delta \varphi d x d t+\lambda \iint_{Q_{T}}|u|^{p-2} u \varphi d x d t=0 .
$$

3) $u(x, 0)=u_{0}(x)$ in $L^{2}(\Omega)$.

This paper is organized as follows. We first discuss the existence of weak solutions in Section 2. Our method is based on the discrete-time method to construct approximate solutions. By means of uniform estimates on solutions of the time-difference equations, we prove the existence of weak solutions of the problem (1.1)-(1.3). Using energy techniques, the Poincaré inequality and Hardy inequality, we also prove the asymptotic behavior and finite speed of propagation of perturbations.

2. Existence of weak solutions. In this section, we prove

Theorem 2.1. Let $u_{0} \in W_{0}^{2, p}(\Omega), p>2$. Then the problem (1.1)-(1.3) admits at least one weak solution.

We first consider the following discrete-time problem:

$$
\frac{1}{h}\left(u_{k+1}-u_{k}\right)+\Delta\left(\left|\Delta u_{k+1}\right|^{p-2} \Delta u_{k+1}\right)+\lambda\left|u_{k+1}\right|^{p-2} u_{k+1}=0,
$$




$$
\left.u_{k+1}\right|_{\partial \Omega}=\left.\Delta u_{k+1}\right|_{\partial \Omega}=0, \quad k=0,1, \ldots, N-1,
$$

where $h=T / N, u_{0}$ is the initial value.

Lemma 2.1. For any fixed $k$, if $u_{k} \in L^{2}(\Omega)$, then the problem (2.1)-(2.2) admits a weak solution $u_{k+1} \in W_{0}^{2, p}(\Omega)$ such that for any $\varphi \in C_{0}^{\infty}(\Omega)$,

$$
\begin{aligned}
\frac{1}{h} \int_{\Omega}\left(u_{k+1}-u_{k}\right) \varphi d x+\int_{\Omega}\left|\Delta u_{k+1}\right|^{p-2} \Delta u_{k+1} \Delta \varphi d x & \\
& +\lambda \int_{\Omega}\left|u_{k+1}\right|^{p-2} u_{k+1} \varphi d x=0 .
\end{aligned}
$$

Proof. Consider the following functionals on the space $W_{0}^{2, p}(\Omega)$ :

$$
\begin{aligned}
& F[u]=\frac{1}{p} \int_{\Omega}|\Delta u|^{p} d x, \\
& G[u]=\frac{1}{2} \int_{\Omega}|u|^{2} d x, \\
& E[u]=\frac{1}{p} \int_{\Omega}|u|^{p} d x, \\
& H[u]=F[u]+\frac{1}{h} G[u]+\lambda E[u]-\int_{\Omega} f u d x,
\end{aligned}
$$

where $f \in L^{2}(\Omega)$ is a known function. By the Young inequality, we see that for $C_{1}>0$,

$$
\begin{aligned}
H[u] & =\frac{1}{p} \int_{\Omega}|\Delta u|^{p} d x+\frac{1}{2 h} \int_{\Omega}|u|^{2} d x+\frac{\lambda}{p} \int_{\Omega}|u|^{p} d x-\int_{\Omega} f u d x \\
& \geq \frac{1}{p} \int_{\Omega}|\Delta u|^{p} d x-C_{1} \int_{\Omega}|f|^{2} d x .
\end{aligned}
$$

We need to check that $H[u]$ satisfies the coercivity condition. For this purpose, we notice that since $\left.u\right|_{\partial \Omega}=0$ and using the $L^{p}$ theory for elliptic equations (see [5]),

$$
\|u\|_{W^{2, p}} \leq C\|\Delta u\|_{L^{p}} .
$$

Therefore $H[u] \rightarrow+\infty$ if $\|u\|_{W^{2, p}} \rightarrow+\infty$. On the other hand, $H[u]$ is clearly weakly lower semicontinuous on $W_{0}^{2, p}(\Omega)$. So, it follows from the theory in [4] that there exists $u_{*} \in W_{0}^{2, p}(\Omega)$ such that

$$
H\left[u_{*}\right]=\inf H[u],
$$

and $u_{*}$ is a weak solution of the Euler equation corresponding to $H[u]$, namely

$$
\frac{1}{h} u+\Delta\left(|\Delta u|^{p-2} \Delta u\right)+\lambda|u|^{p-2} u=f .
$$


Choosing $f=(1 / h) u_{k}$, we get the conclusion of the lemma. The proof is complete.

Now, we construct an approximate solution $u^{h}$ of the problem (1.1)-(1.3) by defining

$$
\begin{aligned}
u^{h}(x, t) & =u_{k}(x), \quad k h<t \leq(k+1) h, k=0,1, \ldots, N-1, \\
u^{h}(x, 0) & =u_{0}(x) .
\end{aligned}
$$

The desired solution of the problem (1.1)-(1.3) will be obtained as the limit of some subsequence of $\left\{u^{h}\right\}$. For this purpose, we need some uniform estimates on $u^{h}$.

Lemma 2.2. For the weak solution $u_{k}$ of the problem (2.1)-(2.2), the following estimates hold:

$$
\begin{gathered}
h \sum_{k=1}^{N} \int_{\Omega}\left|\Delta u_{k}\right|^{p} d x \leq C, \\
\sup _{0<t<T}\left(\int_{\Omega}\left|u^{h}\right|^{p} d x+\int_{\Omega}\left|\Delta u^{h}(x, t)\right|^{p} d x\right) \leq C,
\end{gathered}
$$

where $C$ is a constant independent of $h, k$.

Proof. (i) We take $\varphi=u_{k+1}$ in the integral equality (2.3) (we can easily prove that for $\varphi \in W_{0}^{2, p}(\Omega),(2.3)$ also holds) and obtain

$$
\frac{1}{h} \int_{\Omega}\left|u_{k+1}\right|^{2} d x+\int_{\Omega}\left|\Delta u_{k+1}\right|^{p} d x+\lambda \int_{\Omega}\left|u_{k+1}\right|^{p} d x=\frac{1}{h} \int_{\Omega} u_{k} u_{k+1} d x .
$$

Then by the Young inequality, we have

$$
\begin{aligned}
\frac{1}{h} \int_{\Omega}\left|u_{k+1}\right|^{2} d x+\int_{\Omega}\left|\Delta u_{k+1}\right|^{p} d x+ & \lambda \int_{\Omega}\left|u_{k+1}\right|^{p} d x \\
& \leq \frac{1}{2 h} \int_{\Omega}\left|u_{k}\right|^{2} d x+\frac{1}{2 h} \int_{\Omega}\left|u_{k+1}\right|^{2} d x,
\end{aligned}
$$

i.e.,

$$
\frac{1}{2} \int_{\Omega}\left|u_{k+1}\right|^{2} d x+h \int_{\Omega}\left|\Delta u_{k+1}\right|^{p} d x+h \lambda \int_{\Omega}\left|u_{k+1}\right|^{p} d x \leq \frac{1}{2} \int_{\Omega}\left|u_{k}\right|^{2} d x .
$$

Summing up these inequalities for $k$ from 0 to $N-1$, we have

$$
h \sum_{k=1}^{N} \int_{\Omega}\left|\Delta u_{k}\right|^{p} d x \leq \int_{\Omega}\left|u_{0}\right|^{2} d x .
$$

So, (2.4) holds.

(ii) We choose $\varphi=u_{k+1}-u_{k}$ in the integral equality (2.3) and integrating by parts, we have 


$$
\begin{aligned}
\frac{1}{h} \int_{\Omega}\left|u_{k+1}-u_{k}\right|^{2} d x+\int_{\Omega}\left|\Delta u_{k+1}\right|^{p-2} \Delta u_{k+1} \Delta\left(u_{k+1}-u_{k}\right) d x & \\
& =-\lambda \int_{\Omega}\left|u_{k+1}\right|^{p-2} u_{k+1}\left(u_{k+1}-u_{k}\right) d x
\end{aligned}
$$

Since the first term on the left hand side of the above equality is nonnegative, it follows that

$$
\begin{aligned}
\lambda \int_{\Omega}\left|u_{k+1}\right|^{p} d x & +\int_{\Omega}\left|\Delta u_{k+1}\right|^{p} d x \\
\leq & \int_{\Omega}\left|\Delta u_{k+1}\right|^{p-2} \Delta u_{k+1} \Delta u_{k} d x+\lambda \int_{\Omega}\left|u_{k+1}\right|^{p-2} u_{k+1} u_{k} d x \\
\leq & \frac{p-1}{p} \int_{\Omega}\left|\Delta u_{k+1}\right|^{p} d x+\frac{1}{p} \int_{\Omega}\left|\Delta u_{k}\right|^{p} d x \\
& +\lambda \frac{p-1}{p} \int_{\Omega}\left|u_{k+1}\right|^{p} d x+\frac{\lambda}{p} \int_{\Omega}\left|u_{k}\right|^{p} d x
\end{aligned}
$$

which implies that

$$
\lambda \int_{\Omega}\left|u_{k+1}\right|^{p} d x+\int_{\Omega}\left|\Delta u_{k+1}\right|^{p} d x \leq \int_{\Omega}\left|\Delta u_{k}\right|^{p} d x+\lambda \int_{\Omega}\left|u_{k}\right|^{p} d x .
$$

For any $m$ with $1 \leq m \leq N-1$, summing up the above inequality for $k$ from 0 to $m-1$, we have

$$
\lambda \int_{\Omega}\left|u_{m}\right|^{p} d x+\int_{\Omega}\left|\Delta u_{m}\right|^{p} d x \leq \int_{\Omega}\left|\Delta u_{0}\right|^{p} d x+\lambda \int_{\Omega}\left|u_{0}\right|^{p} d x .
$$

Hence (2.5) holds.

LEMMA 2.3. Let $u_{k+1}$ be the weak solution of the problem (2.1)-(2.2). Then the following estimate holds:

$$
-C h \leq \int_{\Omega}\left|u_{k+1}\right|^{2} d x-\int_{\Omega}\left|u_{k}\right|^{2} d x \leq 0,
$$

where $C$ is a constant independent of $h$.

Proof. To prove the first inequality, we choose $\varphi=u_{k}$ in (2.3), and integrating by parts and using the boundary condition, we obtain

$$
\begin{aligned}
\frac{1}{h} \int_{\Omega}\left|u_{k}\right|^{2} d x= & \frac{1}{h} \int_{\Omega} u_{k+1} u_{k} d x+\int_{\Omega}\left|\Delta u_{k+1}\right|^{p-2} \Delta u_{k+1} \Delta u_{k} d x \\
& +\lambda \int_{\Omega}\left|u_{k+1}\right|^{p-2} u_{k+1} u_{k} d x .
\end{aligned}
$$


Applying the Hölder inequality and the estimate (2.5), we have

$$
\begin{aligned}
\frac{1}{h} \int_{\Omega}\left|u_{k}\right|^{2} d x \leq & \frac{1}{h} \int_{\Omega} u_{k+1} u_{k} d x+\frac{p-1}{p} \int_{\Omega}\left|\Delta u_{k+1}\right|^{p}+\frac{1}{p} \int_{\Omega}\left|\Delta u_{k}\right|^{p} d x \\
& +\lambda \frac{p-1}{p} \int_{\Omega}\left|u_{k+1}\right|^{p} d x+\frac{\lambda}{p} \int_{\Omega}\left|u_{k}\right|^{p} d x \\
\leq & \frac{1}{2 h} \int_{\Omega}\left|u_{k+1}\right|^{2} d x+\frac{1}{2 h} \int_{\Omega}\left|u_{k}\right|^{2} d x+C,
\end{aligned}
$$

that is,

$$
-C h \leq \int_{\Omega}\left|u_{k+1}\right|^{2} d x-\int_{\Omega}\left|u_{k}\right|^{2} d x .
$$

By (2.6) again, we have

$$
\int_{\Omega}\left|u_{k+1}\right|^{2} d x-\int_{\Omega}\left|u_{k}\right|^{2} d x \leq 0 .
$$

The proof is complete.

Proof of Theorem 2.1. First, we define the operator $A^{t}$ by $A^{t}\left(\Delta u^{h}\right)=$ $\left|\Delta u_{k}\right|^{p-2} \Delta u_{k}, \Delta^{h} u^{h}=u_{k+1}-u_{k}$, where $k h<t \leq(k+1) h, k=0,1, \ldots$, $N-1$. From the discrete equation (2.1) and (2.4) in Lemma 2.2, we see that

$$
\frac{1}{h} \Delta^{h} u^{h} \text { is bounded in } L^{\infty}\left(0, T ;\left(W^{2, p}(\Omega)\right)^{\prime}\right) .
$$

By (2.3), (2.5), (2.8) and using compactness results (see [8]), we see that there exists a subsequence of $\left\{u^{h}\right\}$ (which we denote as the original sequence) such that

$$
\begin{aligned}
u^{h} \stackrel{\star}{\rightarrow} u & \text { in } L^{\infty}\left(0, T ; W^{2, p}(\Omega)\right), \\
u^{h} \rightarrow u & \text { in } C\left(0, T ; L^{2}(\Omega)\right), \\
\frac{1}{h}\left(u_{k+1}-u_{k}\right) \stackrel{\star}{\star} \frac{\partial u}{\partial t} & \text { in } L^{\infty}\left(0, T ;\left(W^{2, p}(\Omega)\right)^{\prime}\right), \\
A^{t}\left(\Delta u^{h}\right) \stackrel{\star}{*} w & \text { in } L^{\infty}\left(0, T ; L^{p^{\prime}}(\Omega)\right),
\end{aligned}
$$

where $p^{\prime}$ is the conjugate exponent of $p$. Then from (2.3), we see that, for any $\varphi \in C_{0}^{\infty}\left(Q_{T}\right)$,

$$
\iint_{Q_{T}}\left(\frac{1}{h} \Delta^{h} u^{h} \varphi+A^{t}\left(\Delta u^{h}\right) \Delta \varphi+\lambda\left|u^{h}\right|^{p-2} u^{h} \varphi\right) d x d t=0 .
$$

Letting $h \rightarrow 0$ yields

$$
\frac{\partial u}{\partial t}+\Delta w+\lambda|u|^{p-2} u=0
$$

in the sense of distributions. 
It remains to prove that $w=|\Delta u|^{p-2} \Delta u$ a.e. in $Q_{T}$. Set

$$
f_{h}(t)=\frac{t-k h}{2 h}\left(\int_{\Omega}\left|u_{k+1}\right|^{2} d x-\int_{\Omega}\left|u_{k}\right|^{2} d x\right)+\frac{1}{2} \int_{\Omega}\left|u_{k}\right|^{2} d x,
$$

where $k h<t \leq(k+1) h, k=0,1, \ldots, N-1$. By $(2.7)$, we have

$$
\frac{1}{2} \int_{\Omega}\left|u_{k}\right|^{2} d x-C h \leq f_{h}(t) \leq \frac{1}{2} \int_{\Omega}\left|u_{k}\right|^{2} d x,
$$

and

$$
-C \leq f_{h}^{\prime}(t) \leq 0
$$

According to the Ascoli-Arzelà theorem, there exists a function $f(t) \in$ $C([0, T])$ such that

$$
\lim _{h \rightarrow 0} f_{h}(t)=f(t) \quad \text { uniformly for } t \in[0, T] .
$$

Using (2.7), we have

$$
\lim _{h \rightarrow 0} \frac{1}{2} \int_{\Omega}\left|u^{h}\right|^{2} d x=f(t) \quad \text { uniformly for } t \in[0, T] .
$$

It follows from (2.6) that

$$
\frac{1}{2} \int_{\Omega}\left|u_{N}\right|^{2} d x+\iint_{Q_{T}}\left|\Delta u^{h}\right|^{p} d x d t+\lambda \iint_{Q_{T}}\left|u^{h}\right|^{p} d x d t \leq \frac{1}{2} \int_{\Omega}\left|u_{0}\right|^{2} d x .
$$

Letting $h \rightarrow 0$ in the above inequality and using (2.10), we have

$$
\begin{array}{rl}
\lim _{h \rightarrow 0} \iint_{Q_{T}}\left|\Delta u^{h}\right|^{p} & d x d t \\
& \leq f(0)-f(T)=\lim _{\varepsilon \rightarrow 0} \frac{1}{\varepsilon} \int_{0}^{T-\varepsilon}(f(t)-f(t+\varepsilon)) d t \\
& =\lim _{\varepsilon \rightarrow 0} \lim _{h \rightarrow 0} \frac{1}{2 \varepsilon} \int_{0}^{T-\varepsilon} \int_{\Omega}\left(\left|u^{h}(x, t)\right|^{2}-\left|u^{h}(x, t+\varepsilon)\right|^{2}\right) d x d t .
\end{array}
$$

Consider the functional $G[u]=\frac{1}{2} \int_{\Omega}|u|^{2} d x$. Clearly $G[u]$ is convex and $\delta G[u] / \delta u=u$. Thus, we have

$$
\begin{aligned}
\frac{1}{2} \int_{\Omega}\left|u^{h}(x, t)\right|^{2} d x-\frac{1}{2} \int_{\Omega} \mid u^{h}(x, t & +\varepsilon)\left.\right|^{2} d x \\
& \leq \int_{\Omega}\left(u^{h}(x, t)-u^{h}(x, t+\varepsilon)\right) u^{h}(x, t) d x .
\end{aligned}
$$


Thus

$$
\begin{array}{r}
\lim _{h \rightarrow 0} \frac{1}{2 \varepsilon} \int_{0}^{T-\varepsilon} \int_{\Omega}\left(\left|u^{h}(x, t)\right|^{2}-\left|u^{h}(x, t+\varepsilon)\right|^{2}\right) d x d t \\
\leq \frac{1}{\varepsilon} \int_{0}^{T-\varepsilon} \int_{\Omega}(u(x, t)-u(x, t+\varepsilon)) u d x d t
\end{array}
$$

hence

$$
\lim _{h \rightarrow 0} \iint_{Q_{T}}\left|\Delta u^{h}\right|^{p} d x d t \leq-\int_{0}^{T}\left\langle\frac{\partial u}{\partial t}, u\right\rangle d t
$$

where $\langle\cdot, \cdot\rangle$ denotes the inner product. From $(2.9)$, we have

$$
\lim _{h \rightarrow 0} \iint_{Q_{T}}\left|\Delta u^{h}\right|^{p} d x d t \leq \int_{0}^{T} \int_{\Omega} w \Delta u d x d t+\lambda \int_{0}^{T} \int_{\Omega}|u|^{p} d x d t
$$

Again since $\left.\delta F[u] / \delta u=\Delta\left(|\Delta u|^{p-2} \Delta u\right)\right)$ and by the convexity of $F[u]$, for any $g \in L^{\infty}\left(0, T ; W_{0}^{2, p}(\Omega)\right)$ we have

$$
\frac{1}{p} \iint_{Q_{T}}|\Delta g|^{p} d x d t-\frac{1}{p} \iint_{Q_{T}}\left|\Delta u^{h}\right|^{p} d x d t \geq \iint_{Q_{T}}\left(\left|\Delta u^{h}\right|^{p-2} \Delta u^{h}\right) \Delta\left(g-u^{h}\right) d x d t .
$$

By (2.11) and the fact that $F(u)$ is weakly lower semicontinuous, letting $h \rightarrow 0$ in the above equality, we have

$$
\frac{1}{p} \iint_{Q_{T}}|\Delta g|^{p} d x d t-\frac{1}{p} \iint_{Q_{T}}|\Delta u|^{p} d x d t \geq-\iint_{Q_{T}} w \Delta(u-g) d x d t .
$$

Replacing $g$ by $\varepsilon g+u$, we see that

$$
\frac{1}{\varepsilon}(F[u+\varepsilon g]-F[u]) \geq \iint_{Q_{T}} w \Delta g d x d t .
$$

Letting $\varepsilon \rightarrow 0$ implies that

$$
\iint_{Q_{T}} \frac{\delta F[u]}{\delta u} g d x d t=\iint|\Delta u|^{p-2} \Delta u \Delta g d x d t \geq \iint_{Q_{T}} w \Delta g d x d t .
$$

Due to the arbitrariness of $g$, we also get the opposite inequality to the above inequality. Therefore

$$
w=|\Delta u|^{p-2} \Delta u
$$

The strong convergence of $u^{h}$ in $C\left(0, T ; L^{2}(\Omega)\right)$ and the fact that $u^{h}(x, 0)$ $=u_{0}(x)$ imply that $u$ satisfies the initial condition. The proof is complete.

3. Asymptotic behavior. We first show

THEOREM 3.1. The weak solution u obtained in Theorem 2.1 satisfies, for any $0 \leq \varrho \in C^{2}(\bar{\Omega})$, 


$$
\begin{aligned}
& \frac{1}{2} \int_{\Omega} \varrho(x)|u(x, t)|^{2} d x-\frac{1}{2} \int_{\Omega} \varrho(x)\left|u_{0}(x)\right|^{2} d x \\
& \quad=-\iint_{Q_{t}}|\Delta u|^{p-2} \Delta u \Delta(\varrho(x) u(x, \tau)) d x d \tau-\lambda \iint_{Q_{t}} \varrho(x)|u(x, \tau)|^{p} d x d \tau,
\end{aligned}
$$

where $Q_{t}=\Omega \times(0, t)$.

Proof. In the proof of Theorem 2.1, we have

$$
f(t)=\frac{1}{2} \int_{\Omega}|u(x, t)|^{2} d x \in C([0, T]) .
$$

Similarly, we can also easily prove that for any $0 \leq \varrho \in C^{2}(\bar{\Omega})$,

$$
f_{\varrho}(t)=\frac{1}{2} \int_{\Omega} \varrho(x)|u(x, t)|^{2} d x \in C([0, T]) .
$$

Consider the functional

$$
\Phi_{\varrho}[v]=\frac{1}{2} \int_{\Omega} \varrho(x)|v(x)|^{2} d x .
$$

It is easy to see that it is a convex functional on $L^{2}(\Omega)$.

For any $\tau \in(0, T)$ and $h>0$, we have

$$
\Phi_{\varrho}[u(\tau+h)]-\Phi_{\varrho}[u(\tau)] \geq\langle u(\tau+h)-u(\tau), \varrho(x) u(x, \tau)\rangle .
$$

Since $\delta \Phi_{\varrho}[v] / \delta v=\varrho(x) v$, for any fixed $t_{1}, t_{2} \in[0, T], t_{1}<t_{2}$, integrating the above inequality with respect to $\tau$ over $\left(t_{1}, t_{2}\right)$, we have

$$
\int_{t_{2}}^{t_{2}+h} \Phi_{\varrho}[u(\tau)] d \tau-\int_{t_{1}}^{t_{1}+h} \Phi_{\varrho}[u(\tau)] d \tau \geq \int_{t_{1}}^{t_{2}}\langle u(\tau+h)-u(\tau), \varrho(x) u\rangle d \tau .
$$

Multiplying both sides of the above inequality by $1 / h$, and letting $h \rightarrow 0$, we obtain

$$
\Phi_{\varrho}\left[u\left(t_{2}\right)\right]-\Phi_{\varrho}\left[u\left(t_{1}\right)\right] \geq \int_{t_{1}}^{t_{2}}\left\langle\frac{\partial u}{\partial t}, \varrho(x) u\right\rangle d \tau .
$$

Similarly, we have

$$
\Phi_{\varrho}[u(\tau)]-\Phi_{\varrho}[u(\tau-h)] \leq\langle(u(\tau)-u(\tau-h)), \varrho(x) u\rangle .
$$

Thus

$$
\Phi_{\varrho}\left[u\left(t_{2}\right)\right]-\Phi_{\varrho}\left[u\left(t_{1}\right)\right] \leq \int_{t_{1}}^{t_{2}}\left\langle\frac{\partial u}{\partial t}, \varrho(x) u\right\rangle d \tau
$$

and hence

$$
\Phi_{\varrho}\left[u\left(t_{2}\right)\right]-\Phi_{\varrho}\left[u\left(t_{1}\right)\right]=\int_{t_{1}}^{t_{2}}\left\langle\frac{\partial u}{\partial t}, \varrho(x) u\right\rangle d \tau
$$


Taking $t_{1}=0, t_{2}=t$, from the definition of solutions we get

$$
\begin{aligned}
& \Phi_{\varrho}[u(t)]-\Phi_{\varrho}[u(0)]=\int_{0}^{t}\left\langle-\Delta\left(|\Delta u|^{p-2} \Delta u\right)-\lambda|u|^{p-2} u, \varrho(x) u(\tau)\right\rangle d \tau \\
&=-\int_{0}^{t}\left\langle|\Delta u|^{p-2} \Delta u, \Delta[\varrho(x) u(\tau)]\right\rangle d \tau-\int_{0}^{t}\left\langle\lambda|u|^{p-2} u, \varrho(x) u(\tau)\right\rangle d \tau .
\end{aligned}
$$

TheOrem 3.2. Let $u$ be the weak solution of the problem (1.1)-(1.3), $p>2$. Then

$$
\int_{\Omega}|u(x, t)|^{2} d x \leq \frac{C_{3}}{\left(C_{1} t+C_{2}\right)^{\alpha}}, \quad C_{i}>0(i=1,2,3), \alpha=\frac{2}{p-2} .
$$

Proof. Taking $\varrho(x)=1$ in the equality (3.1), we have

$$
\frac{1}{2} \int_{\Omega}|u(x, t)|^{2} d x-\frac{1}{2} \int_{\Omega}\left|u_{0}(x)\right|^{2} d x=-\int_{0}^{t} \int_{\Omega}|\Delta u|^{p} d x d t-\lambda \iint_{Q_{t}}|u|^{p} d x d t .
$$

Let $f(t)=\frac{1}{2} \int_{\Omega}|u(x, t)|^{2} d x$. By (3.2), we have

$$
f^{\prime}(t)=-\int_{\Omega}|\Delta u|^{p} d x-\lambda \int_{\Omega}|u|^{p} d x \leq 0 .
$$

Since $u \in W_{0}^{2, p}(\Omega)$ and using the Poincaré inequality, we see that

$$
\int_{\Omega}|u(x, t)|^{2} d x \leq C \int_{\Omega}|\Delta u|^{2} d x \leq C\left(\int_{\Omega}|\Delta u|^{p} d x\right)^{2 / p},
$$

that is, $f(t) \leq C\left|f^{\prime}(t)\right|^{2 / p}$.

Again since $f^{\prime}(t) \leq 0$, we have $f^{\prime}(t) \leq-C f(t)^{p / 2}$, and hence

$$
\int_{\Omega}|u(x, t)|^{2} d x \leq \frac{1}{\left(C_{1} t+C_{2}\right)^{\alpha}}, \quad \alpha=\frac{2}{p-2}, C_{i}>0, i=1,2 .
$$

The proof is complete.

\section{Finite speed of propagation of solutions}

TheOREM 4.1. Assume $p>2,\left|\sigma_{n}(0)\right| \leq b$, and $u$ is the weak solution of the problem (1.1)-(1.3). Then for any fixed $t>0$, we have

$$
\sigma_{n}(t)-\sigma_{n}(0) \leq C t^{\alpha}\left(\int_{0}^{t} \int_{\Omega}|\Delta u|^{p} d x d t\right)^{\beta}
$$

where $C$ is constant depending on $p, n, b ; \sigma_{n}(t)=\sup \{z: x \in \operatorname{supp} u(\cdot, t)\}$, $z=x_{n} ; \alpha>0, \beta>0, b>0$ are constants independent of $t$.

To prove Theorem 4.1, we need the following lemma. 
LEMMA $4.1([3])$. Let $f_{s}(z)=\int_{z}^{\infty}(x-z)^{s} g(x) d x, 0 \leq g \in L^{1}\left(\mathbb{R}_{+}\right), k>0$, $\alpha>0, \theta>0, s \geq 1$, and $0<h \leq s<w=\theta h /(\theta-1)$. Assume $f_{s-h}(0)$ is finite and

$$
f_{s}(z) \leq k^{\alpha}\left(f_{s-h}(z)\right)^{\theta}, \quad \forall z \geq 0
$$

Then the support of $f_{0}$ is a bounded interval $[0, l]$ and

$$
l \leq(w-s+1) k^{\alpha /(\theta-1)(w-s)} f_{0}(0)^{1 /(w-s)} .
$$

Proof of Theorem 4.1 Without loss of generality, we assume $\sigma_{n}(t)>0$. By $(3.1)$, taking $\varrho(z)=\left(z-z_{0}\right)_{+}^{s}, z_{0} \geq b, s \geq 2 p$, we have

$$
\begin{aligned}
& \frac{1}{2} \int_{\Omega}\left(z-z_{0}\right)_{+}^{s}|u(x, t)|^{2} d x \\
& \quad=-\int_{0}^{t} \int_{\Omega}|\Delta u|^{p-2} \Delta u \Delta\left[\left(z-z_{0}\right)_{+}^{s} u\right] d x d \tau-\lambda \iint_{Q_{t}}\left(z-z_{0}\right)_{+}^{s}|u(\tau)|^{p} d x d \tau .
\end{aligned}
$$

Denote the left side of the above equality by $I$. Then we have

$$
\begin{aligned}
I= & -\int_{0}^{t} \int_{\Omega}|\Delta u|^{p-2} \Delta u \Delta\left[\left(z-z_{0}\right)_{+}^{s} u\right] d x d \tau-\lambda \iint_{Q_{t}}\left(z-z_{0}\right)_{+}^{s}|u(\tau)|^{p} d x d \tau \\
= & -\int_{0}^{t} \int_{\Omega}\left(z-z_{0}\right)_{+}^{s}|\Delta u|^{p} d x d \tau-2 \iint_{0}^{t} \nabla\left[\left(z-z_{0}\right)_{+}^{s}\right] \nabla u|\Delta u|^{p-2} \Delta u d x d \tau \\
& -\int_{0}^{t} \int_{\Omega} s(s-1)\left(z-z_{0}\right)_{+}^{s-2} u|\Delta u|^{p-2} \Delta u d x d \tau-\lambda \iint_{Q_{t}}\left(z-z_{0}\right)_{+}^{s}|u(\tau)|^{p} d x d \tau .
\end{aligned}
$$

By the Hölder inequality,

$$
\begin{aligned}
I \leq & -\int_{0}^{t} \int_{\Omega}\left(z-z_{0}\right)_{+}^{s}|\Delta u|^{p} d x d \tau+\frac{1}{4} \iint_{0}^{t}\left(z-z_{0}\right)_{+}^{s}|\Delta u|^{p} d x d \tau \\
& +C_{1} \int_{0}^{t} \int_{\Omega}\left(z-z_{0}\right)_{+}^{s-p}|\nabla u|^{p} d x d \tau+\frac{1}{4} \int_{0}^{t}|\Delta u|_{\Omega}^{p}\left(z-z_{0}\right)_{+}^{s} d x d \tau \\
& +C_{2} \int_{0}^{t} \int_{\Omega}\left(z-z_{0}\right)_{+}^{s-2 p}|u|^{p} d x d \tau-\lambda \int_{0}^{t} \int_{\Omega}\left(z-z_{0}\right)_{+}^{s}|u(\tau)|^{p} d x d \tau \\
\leq & -\frac{1}{2} \int_{0}^{t} \int_{\Omega}\left(z-z_{0}\right)_{+}^{s}|\Delta u|^{p} d x d \tau+C_{1} \int_{0} \int_{\Omega}\left(z-z_{0}\right)_{+}^{s-p}|\nabla u|^{p} d x d \tau \\
& +C_{2} \int_{0}^{t} \int_{\Omega}\left(z-z_{0}\right)_{+}^{s-2 p}|u|^{p} d x d \tau .
\end{aligned}
$$


Applying the Hardy inequality [7], we obtain

$$
\int_{\Omega}\left(z-z_{0}\right)_{+}^{s-2 p}|u|^{p} d x \leq\left(\frac{p}{s-2 p+1}\right)^{p} \int_{\Omega}\left(z-z_{0}\right)_{+}^{s-p}\left|D_{z} u\right|^{p} d x .
$$

Hence

$$
\begin{aligned}
& \frac{1}{2} \int_{\Omega}\left(z-z_{0}\right)_{+}^{s}|u|^{2} d x+\frac{1}{2} \int_{0}^{t} \int_{\Omega}\left(z-z_{0}\right)_{+}^{s}|\Delta u|^{p} d x d \tau \\
& \quad \leq C_{3} \int_{0}^{t} \int_{\Omega}\left(z-z_{0}\right)_{+}^{s-p}|\nabla u|^{p} d x d \tau+C_{4} \int_{0}^{t} \int_{\Omega}\left(z-z_{0}\right)_{+}^{s-p}\left|D_{z} u\right|^{p} d x d \tau \\
& \quad \leq C \int_{0}^{t} \int_{\Omega}\left(z-z_{0}\right)_{+}^{s-p}|\nabla u|^{p} d x d \tau
\end{aligned}
$$

Thus

$$
\sup _{0<\tau \leq t} \int_{\Omega}\left(z-z_{0}\right)_{+}^{s}|u|^{2} d x \leq C \iint_{Q_{t}}\left(z-z_{0}\right)_{+}^{s-p}|\nabla u|^{p} d x d \tau
$$

and

$$
\iint_{Q_{t}}\left(z-z_{0}\right)_{+}^{s}|\Delta u|^{p} d x d \tau \leq C \iint_{Q_{t}}\left(z-z_{0}\right)_{+}^{s-p}|\nabla u|^{p} d x d \tau .
$$

From (4.2) again using the Hardy inequality, we have

$$
\sup _{0<\tau \leq t} \int_{\Omega}\left(z-z_{0}\right)_{+}^{s}|u|^{2} d x \leq C \iint_{Q_{t}}\left(z-z_{0}\right)_{+}^{s}|\Delta u|^{p} d x d \tau .
$$

Set

$$
E_{s}\left(z_{0}\right)=\iint_{Q_{t}}\left(z-z_{0}\right)_{+}^{s}|\Delta u|^{p} d x d \tau, \quad E_{0}\left(z_{0}\right)=\int_{0}^{t} \int_{\Omega}|\Delta u|^{p} d x d \tau .
$$

From (4.3) and the weighted Nirenberg inequality, we have

$$
\begin{aligned}
E_{2 p+1}\left(z_{0}\right) \leq & C_{1} \iint_{Q_{t}}\left(z-z_{0}\right)_{+}^{p+1}|\nabla u|^{p} d x d \tau \\
& \leq C \int_{0}^{t}\left(\int_{\Omega}\left(z-z_{0}\right)_{+}^{p+1}|\Delta u|^{p} d x\right)^{a}\left(\int_{\Omega}\left(z-z_{0}\right)_{+}^{p+1}|u|^{2} d x\right)^{(1-a) p / 2} d \tau,
\end{aligned}
$$

where

$$
\frac{1}{p}=\frac{1}{p+2}+a\left(\frac{1}{p}-\frac{2}{p+2}\right)+(1-a) \frac{1}{2}
$$

therefore

$$
a=\frac{\frac{1}{p}-\frac{1}{p+2}-\frac{1}{2}}{\frac{1}{p}-\frac{2}{p+2}-\frac{1}{2}}<1 .
$$


Using (4.4) we obtain

$$
\begin{aligned}
E_{2 p+1}\left(z_{0}\right) \leq & C\left(\iint_{Q_{t}}\left(z-z_{0}\right)_{+}^{p+1}|\Delta u|^{p} d x d \tau\right)^{(1-a) p / 2} \\
& \times \int_{0}^{t} \int_{\Omega}\left(\left(z-z_{0}\right)_{+}^{p+1}|\Delta u|^{p} d x\right)^{a} d \tau \\
\leq & C\left[E_{p+1}\left(z_{0}\right)\right]^{(1-a) p / 2}\left(\iint_{Q_{t}}\left(z-z_{0}\right)_{+}^{p+1}|\Delta u|^{p} d x d \tau\right)^{a} t^{1-a} \\
\leq & C E_{p+1}\left(z_{0}\right)^{(1-a) p / 2+a} t^{1-a} .
\end{aligned}
$$

From the above inequality we obtain $\Delta u=0$ a.e. for $z_{0}>b$ and $0<\tau$ $<t$. By (4.4), we know that $u=0$ a.e. on the same set. By Lemma 4.1, we obtain Theorem 4.1. The proof is complete.

\section{References}

[1] J. Benedikt, Uniqueness theorem for p-biharmonic equations, Electron. J. Differential Equations 2002, no. 53, 17 pp.

[2] -, On simplicity of spectra of p-biharmonic equations, Nonlinear Anal. 58 (2004), 835-853.

[3] F. Bernis, Qualitative properties for some nonlinear higher order degenerate parabolic equations, Houston J. Math. 14 (1988), 319-352.

[4] K. Chang, Critical Point Theory and its Applications, Shanghai Sci. Tech. Press, Shanghai, 1986.

[5] Y. Chen and L. Wu, Second Order Elliptic Equations and Elliptic Systems, Science Press, Beijing, 1991.

[6] P. Drábek and M. Ôtani, Global bifurcation result for the p-biharmonic operator, Electron. J. Differential Equations 2001, no. 48, 19 pp.

[7] G. H. Hardy, J. E. Littlewood and G. Pólya, Inequalities, Cambridge Univ. Press, Cambridge, 1952.

[8] J. Simon, Compact sets in the space $L^{p}(0, T ; B)$, Ann. Mat. Pura Appl. 146 (1987), $65-96$.

Changchun Liu

Department of Mathematics

Jilin University

Changchun 130012, P.R. China

E-mail: liucc@jlu.edu.cn
Jinyong Guo

Department of Mathematics

Liuzhou Normal College Liuzhou, 545004, P.R. China

Received February 20, 2006;

received in final form June 20, 2006 